Pacific Journal of Mathematic 


\section{WEIGHTS INDUCED BY HOMOGENEOUS POLYNOMIALS}

\section{Boo Rim Choe}

Let $B$ be the unit ball and $S$ the unit sphere in $\mathbb{C}^{n}(n \geq 2)$. Let $\sigma$ be the unique normalized rotation-invariant Borel measure on $S$ and $m$ the normalized area measure on $C$.

We first prove that if $\Lambda$ is a holomorphic homogeneous polynomial on $\mathbb{C}^{n}$ normalized so that $\Lambda$ maps $B$ onto the unit disk $U$ in $\mathbb{C}$ and if $\mu=\sigma\left[\left(\left.\Lambda\right|_{S}\right)^{-1}\right]$, then $\mu \ll m$ and the Radon-Nikodym derivative $d \mu / d m$ is radial and positive on $U$. Then we obtain the asymptotic behavior of $d \mu / d m$ for a certain, but not small, class of functions $\Lambda$. These results generalize two recent special cases of $P$. Ahern and $P$. Russo. As an immediate consequence we enlarge the class of functions for which Ahern-Rudin's Paley-type gap theorems hold.

1. Introduction. Let $n$ be a positive integer. Write $B=B_{n}$ for the unit ball in $\mathbb{C}^{n}$ and let $S=S_{n}=\partial B_{n}$. When $n=1$, we use the notation $U$ and $T$ in place of $B_{1}$ and $S_{1}$, respectively. We shall let $\sigma=\sigma_{n}$ denote the unique normalized rotation-invariant Borel measure on $S$ and $m$ the normalized area measure on $\mathbb{C}$. The symbol $P_{n}$ stands for the class of holomorphic homogeneous polynomials $\Lambda$ on $\mathbb{C}^{n}$ normalized so that $\Lambda(B)=U$. The maximum modulus set $\Lambda^{-1}(T) \cap S$ of $\Lambda \in P_{n}$ is denoted by $\operatorname{Max} \Lambda$. It is assumed $n \geq 2$ in the rest of the paper unless otherwise specified.

To begin with, let us look at some special cases which motivated the main results of this paper. We have the following "change-ofvariables" formula for $\Lambda \in P_{n}$ of degree 1 [Ru, Section 1.4]:

$$
\int_{S} \psi \circ \Lambda^{*} d \sigma=(n-1) \int_{U} \psi(\lambda)\left(1-|\lambda|^{2}\right)^{n-2} d m(\lambda) \text {. }
$$

Here $\Lambda^{*}=\left.\Lambda\right|_{S}$ and $\psi$ denotes an arbitrary nonnegative Borel function on $U$. The similar integral formula for $\Lambda(z)=z_{1}^{2}+\cdots+z_{n}^{2}$ has been recently proved by $\mathbf{P}$. Russo [Rus]:

$$
\int_{S} \psi \circ \Lambda^{*} d \sigma=(n-1) / 2 \int_{U} \psi(\lambda)\left(1-|\lambda|^{2}\right)^{(n-3) / 2} d m(\lambda) .
$$

Also, P. Ahern [A] has shown that if $\Lambda(z)=n^{n / 2} z_{1} \cdots z_{n}$, then

$$
\int_{S} \psi \circ \Lambda^{*} d \sigma=\int_{U} \psi(\lambda) w(\lambda) d m(\lambda)
$$


where $w$ is a weight such that

$$
0<\lim _{r \uparrow 1} w(r) /\left(1-r^{2}\right)^{(n-3) / 2}<\infty .
$$

Here and in what follows, a weight means a positive $[m]$ a.e., radial, integrable Borel function on $U$.

Formula (1) is an immediate consequence of Formula 1.4.4 in [Ru]. In [A] and [Rus], while (2) and (3) are the important first steps to the main pullback results (see 3.4 below), their proofs strongly depend on the precise formula of $\Lambda$. In $\S 2$ we prove that every $\Lambda \in P_{n}$ induces a unique (up to a set of $[m]$ measure 0 ) weight $w$ so that (3) holds. Note that the exponents in (2) and (4) are the same, while they are different from that in (1). Why? One possible way to explain this might be as follows. It is easily verified that if $\Lambda(z)=z_{1}^{2}+\cdots+z_{n}^{2}$, then $\operatorname{Max} \Lambda$ is topologically equivalent to $S^{n-1} \times T$ and if $\Lambda(z)=n^{n / 2} z_{1} \cdots z_{n}$, then $\operatorname{Max} \Lambda$ is topologically equivalent to $T^{n}$. Also it is clear that if $\Lambda \in P_{n}$ is of degree 1 , then $\operatorname{Max} \Lambda=T$, topologically. Hence a close look at the exponents in (1), (2) and (4) leads to the interesting fact that all these exponents can be written as a single expression $\delta(\Lambda) / 2-1$. The notation $\delta(\Lambda)$ means the topological co-dimension of Max $\Lambda$ in $S$, i.e., $\delta(\Lambda)=(2 n-1)-\operatorname{dim}(\operatorname{Max} \Lambda)$ where $\operatorname{dim}(\operatorname{Max} \Lambda)$ denotes the topological dimension of $\operatorname{Max} \Lambda$. It is known that $\delta(\Lambda) \geq n-1$ (see [Ru, Section 11.4]). Although one cannot expect precise formulas of weights as in (1) and (2) in general, it is a pleasant fact that $\delta(\Lambda) / 2-1$ is the right exponent for a certain class, say $\Omega_{n}$, of functions $\Lambda \in P_{n}$. More precisely, in $\S 3$ it is shown that if $w$ is the weight induced by $\Lambda \in \Omega_{n}$ in the sense of (3), then

$$
w(r)=c(\Lambda)\left(1-r^{2}\right)^{\delta(\Lambda) / 2-1}\left[1+O\left(\sqrt{1-r^{2}}\right)\right] \quad \text { as } r \uparrow 1 .
$$

Even when $n=1, \delta(\Lambda) / 2-1$ is the right exponent at least in the weak sense. Of course $\delta(\Lambda)=0$ for every $\Lambda \in P_{1}$ and it is not hard to verify that

$$
\lim _{\alpha \downarrow 0} \alpha\left(1-|\lambda|^{2}\right)^{\alpha-1} d m(\lambda)=\frac{d \theta}{2 \pi}
$$

in the weak*-topology of the dual of $C(\bar{U})$. Hence

$$
\begin{aligned}
\int_{0}^{2 \pi} \psi \circ \Lambda\left(e^{i \theta}\right) \frac{d \theta}{2 \pi} & =\int_{0}^{2 \pi} \psi\left(e^{i \theta}\right) \frac{d \theta}{2 \pi} \\
& =\lim _{\alpha \downarrow 0} \alpha \int_{\bar{U}} \psi(\lambda)\left(1-|\lambda|^{2}\right)^{\alpha-1} d m(\lambda)
\end{aligned}
$$

for every $\psi \in C(\bar{U})$.

We do not know whether $(*)$ holds for every $\Lambda \in P_{n}$. 
Acknowledgement. This paper represents a part of the author's $\mathrm{Ph} . \mathrm{D}$. thesis. The author would like to thank his advisor W. Rudin for helpful suggestions.

2. Existence. In this section we shall prove that to each $\Lambda \in P_{n}$ corresponds a unique weight as mentioned above. This will be derived from a more general fact Theorem 2.4 below. We shall let $\langle$,$\rangle denote$ the complex inner product on $\mathbb{C}^{n}$ and $|z|^{2}=\langle z, z\rangle$ for $z \in \mathbb{C}^{n}$.

2.1. Jacobian and co-area formula. Consider a continuously differentiable function $\phi: S \rightarrow \mathbb{C}$. Fix $\xi \in S$ and $T_{\xi}(S)$ be the real tangent space to $S$ at $\xi$. We regard $T_{\xi}(S)$ as a $(2 n-1)$-dimensional real space sitting in $\mathbb{C}^{n}$. Also $\mathbb{C}$ is regarded as a 2-dimensional real space. Therefore, for $z \in \mathbb{C}^{n}, z \in T_{\xi}(S)$ if and only if $\operatorname{Re}\langle\xi, z\rangle=0$. Let $D$ be the differential of $\phi$ at $\xi$ defined by

$$
D(X)=\left.\frac{d}{d t} \phi(\gamma(t))\right|_{t=0} \text { for } X \in T_{\xi}(S)
$$

where $\gamma$ is any curve in $S$ such that $\gamma(0)=\xi$ and $\gamma^{\prime}(0)=X$. If rank $D \leq 1$, then $\xi$ is called a critical point of $\phi$. The Jacobian of $\phi$ at $\xi$ is defined by

$$
J \phi(\xi)=\sqrt{\operatorname{det} D \circ D^{*}} .
$$

By the Binet-Cauchy formula [G], one computes $J \phi(\xi)$ as the square root of the sum of squares of the 2 by 2 subdeterminants of the matrix realization of $D$ with respect to orthonormal bases of $T_{\xi}(S)$ and $\mathbb{C}$. It follows that $\xi$ is a critical point of $\phi$ if and only if $J \phi(\xi)=0$.

Federer's co-area formula for manifolds [F1, Theorem 3.1] will be the key to proving the existence of weights. A simple version, which is enough for our purpose, is as follows:

$$
\int_{S} g J \phi d \sigma=c(n) \int_{\mathbb{C}} \int_{\phi^{-1}(\lambda)} g d h_{2 n-3} d m(\lambda)
$$

for every nonnegative Borel function $g$ on $S$. Here $h_{2 n-3}$ denotes the $(2 n-3)$-dimensional Hausdorff measure on $S$.

2.2. Lemma. Let $\phi \in C^{2 n-2}(S)$. Then

$$
\sigma\left[\phi^{-1}\right] \ll m \text { if and only if } J \phi>0[\sigma] \text { a.e. }
$$

Proof. First assume $\sigma\left[\phi^{-1}\right] \ll m$. It suffices to show $m[\phi(K)]=0$ where $K$ is the set of all critical points of $\phi$. But this follows directly from Sard's theorem (see [F2, p. 310]) because $\phi \in C^{2 n-2}(S)$. 
Conversely, assume $J \phi>0[\sigma]$ a.e. By the co-area formula we have

$$
\int_{\phi^{-1}(E)} J \phi d \sigma=c(n) \int_{E} h_{2 n-3}\left[\phi^{-1}(\lambda)\right] d m(\lambda)
$$

for every Borel set $E$ in $\mathbb{C}$. In particular, if $m(E)=0$, then $\sigma\left[\phi^{-1}(E)\right]$ $=0$. In other words, $\sigma\left[\phi^{-1}\right] \ll m$. The proof is complete.

The following lemma was proved for $\Lambda(z)=z_{1}^{2}+\cdots+z_{n}^{2}$ in [Rus]. The proof given below is a modification of P. Russo's proof found there.

2.3. LemMA. Suppose $\phi$ is a function on $S$ which extends to a function holomorphic on some open set containing $\bar{B}$. Then

$$
J \phi=|\nabla \phi| \sqrt{|\nabla \phi|^{2}-|R \phi|^{2}}
$$

where $\nabla \phi$ is the complex gradient of $\phi$ and $R \phi$ is the radial derivative of $\phi$, i.e., $R \phi(\xi)=\langle\nabla \phi(\xi), \bar{\xi}\rangle$.

Proof. Fix $\xi \in S$. Let $D$ be the differential of $\phi$ at $\xi$ and $T_{\xi}(S)$ the real tangent space to $S$ at $\xi$. We may regard $\phi$ as a function holomorphic on some open set containing $\bar{B}$. Hence by the chain rule

$$
D(W)=\langle W, \overline{\nabla \phi}(\xi)\rangle
$$

for every $W \in T_{\xi}(S)$. Define $X=\overline{\nabla \phi}(\xi)-\overline{R \phi}(\xi) \xi$. Then (2) can be written

$$
D(W)=\langle W, X\rangle+i R \phi(\xi) \operatorname{Re}\langle W, i \xi\rangle
$$

because $\operatorname{Re}\langle W, \xi\rangle=0$ for $W \in T_{\xi}(S)$. It is easily seen that $i \xi$, $X$ and $i X$ are orthogonal vectors in $T_{\xi}(S)$. Also it is easy to see $|X|^{2}=|\nabla \phi(\xi)|^{2}-|R \phi(\xi)|^{2}$ because $R \phi(\xi)=\langle\nabla \phi(\xi), \bar{\xi}\rangle$. Using (3), we easily obtain $D(i \xi)=i R \phi(\xi), D(X)=|X|^{2}$ and $D(i X)=i|X|^{2}$. In addition, if $W \in T_{\xi}(S)$ is orthogonal to $i \xi, X$ and $i X$, then $D(W)=0$. Now assume that $X \neq 0$, extend $\{i \xi, X /|X|, i X /|X|\}$ to an ordered orthonormal basis for $T_{\xi}(S)$ and regard $\{1, i\}$ as an ordered orthonormal basis for $\mathbb{C}$. With respect to these orthonormal bases, $D$ can therefore be realized as a 2 by $2 n-1$ matrix

$$
\left(\begin{array}{cccc}
-\operatorname{Im} R \phi(\xi) & |X| & 0 & 0 \cdots 0 \\
\operatorname{Re} R \phi(\xi) & 0 & |X| & 0 \cdots 0
\end{array}\right)
$$

It is easily verified that this matrix realization of $D$ is still valid when $X=0$. A little computation now leads to (1). 
2.4. Theorem. Let $\phi \in C^{2 n-2}(S)$ and $\mu=\sigma\left[\phi^{-1}\right]$. Suppose that $\phi$ is nonconstant and extends to a function holomorphic on $B$. Then

(1) $\mu \ll m$,

(2) $d \mu / d m>0[m]$ a.e. on $\phi(S)$. More explicitly,

$$
\frac{d \mu}{d m}(\lambda)=c(n) \int_{\phi^{-1}(\lambda)} \frac{d h_{2 n-3}}{J \phi} \quad[m] \text { a.e. } \lambda \in \mathbb{C} .
$$

Proof. The proof of Theorem 1 in [S] shows that the set of critical points of $\phi$ has $[\sigma]$ measure 0 . In other words, $J \phi>0[\sigma]$ a.e. Thus by Lemma 2.2, we obtain (1). Since $J \phi$ is also continuous, $\chi_{\phi^{-1}(E)} / J \phi$ is a Borel function on $S$ whenever $E$ is a Borel set in $\mathbb{C}$. Here $\chi_{\phi^{-1}(E)}$ denotes the characteristic function of $\phi^{-1}(E)$. Apply the co-area formula to this function and obtain

$$
\mu(E)=c(n) \int_{E} \int_{\phi^{-1}(\lambda)} \frac{d h_{2 n-3}}{J \phi} d m(\lambda) .
$$

From this it follows that

$$
\frac{d \mu}{d m}(\lambda)=c(n) \int_{\phi^{-1}(\lambda)} \frac{d h_{2 n-3}}{J \phi} \quad[m] \text { a.e. } \lambda \in \mathbb{C} .
$$

Now suppose that $\lambda \in \phi(S)$ is a regular value. This means that $\lambda$ is not in the image of critical points under $\phi$. Since $\lambda$ is a regular value, $\phi^{-1}(\lambda)$ is a $(2 n-3)$-manifold and thus the right side of (3) is strictly positive. Since $\phi \in C^{2 n-2}(S)$, Sard's theorem says that the set of regular values of $\phi$ is of full $[m]$ measure in $\phi(S)$. This proves (2).

We now come to the main result of this section.

2.5. Theorem. Let $\Lambda \in P_{n}$. Then there exists a unique (up to a set of $[\mathrm{m}]$ measure 0$)$ weight $w$ on $U$ such that

$$
\int_{S} \psi \circ \Lambda^{*} d \sigma=\int_{U} \psi w d m
$$

for every nonnegative Borel function $\psi$ on $U$.

Note. Since $\sigma(\operatorname{Max} \Lambda)=0, \psi \circ \Lambda^{*}$ is a Borel function defined [ $\left.\sigma\right]$ a.e. on $S$. This $w$ will be called the weight induced by $\Lambda$.

Proof. Let $\mu=\sigma\left[\left(\Lambda^{*}\right)^{-1}\right]$. By Theorem $2.4 \mu \ll m$. Put $w=$ $d \mu / d m$. Since $\Lambda(S)=\Lambda(\bar{B}), w>0[m]$ a.e. on $U$ by Theorem 2.4. Recall that we have

$$
w(\lambda)=c(n) \int_{\left(\Lambda^{*}\right)^{-1}(\lambda)} \frac{d h_{2 n-3}}{J \Lambda^{*}} \text { for } \lambda \in U
$$


after redefining $w$ on a set of $[m]$ measure 0 . Since $\Lambda \in P_{n},|\nabla \Lambda|$ is $T$ invariant, i.e., $|\nabla \Lambda(\xi)|=\left|\nabla \Lambda\left(\xi e^{i \theta}\right)\right|$ for every $\xi \in S$ and real number $\theta$. Since $R \Lambda=(\operatorname{deg} \Lambda) \Lambda, J \Lambda^{*}$ is $T$-invariant by Lemma 2.3. It follows from homogeneity that $w$ is radial, because $h_{2 n-3}$ is also $T$-invariant. Thus $w$ is a weight on $U$. Now, by definition of $\mu,(1)$ holds for every characteristic function $\psi$ of a Borel set in $U$. The general case follows from a standard approximation. The proof is complete.

2.6. Remark Let $w$ be the weight induced by $\Lambda \in P_{n}$. For every nonnegative Borel function $\psi$ on $[0,1)$ we have

$$
\int_{S} \psi \circ\left|\Lambda^{*}\right| d \sigma=2 \int_{0}^{1} r \psi(r) w(r) d r .
$$

Thus $w$ is completely determined by such $\psi$. This fact will be the basis for the asymptotic estimate of $w$ near $r=1$ in $\S 3$.

3. Estimation. We shall estimate the asymptotic behavior of the weight induced by a function belonging to a subclass of $P_{n}$.

We need some more notation and terminology. Suppose that $\phi_{1}$ and $\phi_{2}$ are $\mathbb{C}$-valued functions on $D_{1} \subset \mathbb{C}^{k}$ and $D_{2} \subset \mathbb{C}^{l}$, respectively. We shall let $\phi_{1} \otimes \phi_{2}$ and $\phi_{1} \oplus \phi_{2}$ denote the functions defined by $\left(\phi_{1} \otimes \phi_{2}\right)(z, w)=\phi_{1}(z) \phi_{2}(w)$ and $\left(\phi_{1} \oplus \phi_{2}\right)(z, w)=\phi_{1}(z)+\phi_{2}(w)$ for $(z, w) \in D_{1} \times D_{2} \subset \mathbb{C}^{k} \times \mathbb{C}^{l}=\mathbb{C}^{k+l}$. For simplicity $\Lambda \in P_{n}$ is called good if Max $\Lambda$ is a disjoint union of finitely many compact manifolds in $S$ and if the weight induced by $\Lambda$ satisfies $(*)$. Also every $\Lambda \in P_{1}$ is called good.

3.1. Theorem. Suppose $\phi_{1} \in P_{k}(k \geq 1)$ and $\phi_{2} \in P_{l}(l \geq 1)$. Let $\Lambda \in P_{n}(n=k+l)$ be one of the following.

(a) $\Lambda=b \phi_{1} \otimes \phi_{2}, b>0$,

(b) $\Lambda=a \phi_{1} \oplus \phi_{2}, \operatorname{deg} \Lambda \geq 3,0 \leq a \leq 1$,

(c) $\Lambda=a \lambda^{2} \oplus \phi_{2}, \operatorname{deg} \Lambda=2,0 \leq a<1$.

In case (c), $k=1$ and $l=n-1$. If $\phi_{1}$ and $\phi_{2}$ are good, then so is $\Lambda$.

3.2. Corollary. Suppose that $\Lambda \in P_{n}$ is a monomial or $\Lambda(z)=$ $a_{1} z_{1}^{d}+\cdots+a_{n} z_{n}^{d}$. Then $\Lambda$ is good.

3.3. Class $\Omega_{n}$. It is easily seen from Remark 2.6 that if $\Lambda$ is good, then so is any power of $\Lambda$. Also, if $\Lambda$ is good, then so is $\Lambda \circ \mathscr{U}$ for every unitary transformation $\mathscr{U}$ on $\mathbb{C}^{n}$ because $\Lambda$ and $\Lambda \circ \mathscr{U}$ induce exactly the same weight and $\operatorname{Max}(\Lambda \circ \mathscr{U})=\mathscr{U}^{*}(\operatorname{Max} \Lambda)$. Now choose any function as in Corollary 3.2, but of fewer variables, apply Theorem 
3.1, (a) through (c), repeatedly to construct a new function in more variables, form any power of this new function, and compose it with any unitary transformation. Let $\Omega_{n}$ be the subclass of $P_{n}$ obtained by any combination of this process. Then every $\Lambda \in \Omega_{n}$ is good. The class $\Omega_{n}$ is not small in the sense that it contains most of those functions which may occur in the actual examples or applications.

Before we prove Theorem 3.1, let us look at some consequences

3.4. Pullback theorem. Let $0<p<\infty$. The Hardy space $H^{p}(B)$ consists of functions $f$ holomorphic on $B$ such that

$$
\|f\|_{p}^{p}=\sup _{0<r<1} \int_{S}|f(r \xi)|^{p} d \sigma(\xi)<\infty .
$$

For $\alpha>-1$, the weighted Bergman space $A_{\alpha}^{p}(U)$ consists of functions $g$ holomorphic on $U$ such that

$$
\int_{U}|g(\lambda)|^{p}\left(1-|\lambda|^{2}\right)^{\alpha} d m(\lambda)<\infty .
$$

Now consider $\Lambda \in \Omega_{n}$ and let $w$ be the weight induced by $\Lambda$. Then $g \in A_{\alpha}^{p}(U)$ where $\alpha=\delta(\Lambda) / 2-1$ if and only if

$$
\int_{U}|g(\lambda)|^{p} w(\lambda) d m(\lambda)<\infty
$$

Hence it follows that $g \circ \Lambda \in H^{p}(B)$ for every $g \in A_{\alpha}^{p}(U)$. This pullback property is sharp in the sense that the "weight" $\delta(\Lambda) / 2-1$ cannot be improved.

It is known [A], [Rus] that if $\Lambda(z)=n^{n / 2} z_{1} \cdots z_{n}$ or $\Lambda(z)=$ $z_{1}^{2}+\cdots+z_{n}^{2}$, then $g \circ \Lambda^{*}$ is a function of bounded mean oscillation on $S$ for every Bloch function $g$ on $U$. P. Ahern and W. Rudin have recently found a new proof of this BMO-result, which does not seem to extend to the $H^{p}$-context mentioned above, and proved the same for every monomial $\Lambda \in P_{n}$. Their method has been used by the author [C] to prove the same BMO-result for a certain subclass of $\Omega_{n}$ containing all the previous functions.

3.5. Gap theorem. Let $E$ be a set of positive integers. Following [AR2] we call $E$ a Paley set if $|\{k \in E: N \leq k \leq 2 N\}|$ stays bounded as $N \rightarrow \infty$. Also we recall that $E$ is called a $\Lambda(q)$-set, for $q>2$, if there is a constant $C$ such that

$$
\left(\int_{0}^{2 \pi}\left|\sum_{k \in E} a_{k} e^{i k \theta}\right|^{q} \frac{d \theta}{2 \pi}\right)^{1 / q} \leq C\left(\sum_{k \in E}\left|a_{k}\right|^{2}\right)^{1 / 2}
$$


for all $\left\{a_{k}\right\}$. It turns out that every Paley set is a $\Lambda(q)$-set for every $q>2$. Fix $\Lambda \in P_{n}$. If $f \in L^{1}(\sigma)$ and if $k$ is a nonnegative integer, then we shall write $f_{k}$ for the projection of $f$ into the one-dimensional space spanned by $\Lambda^{k}$. In their recent paper [AR2] P. Ahern and W. Rudin have also shown the following Paley-type gap theorems which have no analogue in the one variable case for a special class of functions $\Lambda \in P_{n}$, i.e., for $\Lambda$ monomials and $\Lambda(z)=z_{1}^{2}+\cdots+z_{n}^{2}$. We refer to [AR2] for further details:

TheOREM. Let $1<p<2<q<\infty$. Then the following are equivalent.

(a) $E$ is a Paley set.

(b) $\sum_{k \in E}\left\|f_{k}\right\|_{p}^{p} \leq C\|f\|_{p}^{p}$ for every $f \in H^{p}(B)$.

(c) There exists $t<2$ so that $\sum_{k \in E}\left\|f_{k}\right\|_{p}^{t} \leq C\|f\|_{p}^{t}$ for every $f \in$ $H^{p}(B)$.

(d) $\|h\|_{q}^{q} \leq C \sum_{k \in E}\left\|h_{k}\right\|_{q}^{q}$ for every $h \in H^{q}(B)$ of the form $h=$ $\sum_{k \in E} a_{k} \Lambda^{k}$.

(e) There exists $s>2$ so that $\|h\|_{q}^{s} \leq C \sum_{k \in E}\left\|h_{k}\right\|_{q}^{s}$ for every $h \in$ $H^{q}(B)$ of the form $h=\sum_{k \in E} a_{k} \Lambda^{k}$.

THEOREM. Let $1<p<2$ and $q$ the conjugate exponent. If $E$ is a $\Lambda(q)$-set, then the following two equivalent conditions hold.

(a) $\|h\|_{q}^{2} \leq C \sum_{k \in E}\left\|h_{k}\right\|_{q}^{2}$ for every $h \in H^{q}(B)$ of the form $h=$ $\sum_{k \in E} a_{k} \Lambda^{k}$.

(b) $\sum_{k \in E}\left\|f_{k}\right\|_{p}^{2} \leq C\|f\|_{p}^{2}$ for every $f \in H^{p}(B)$.

THEOREM. If $2 \leq p<\infty$, then $\|f\|_{p}^{p} \leq C \sum_{j=0}^{\infty}\left(\sum_{2^{\jmath} \leq k<2^{\jmath+1}}\left\|f_{k}\right\|_{p}\right)^{p}$ holds for every $f \in H^{p}(B)$ of the form $f=\sum_{k=1}^{\infty} a_{k} \Lambda^{k}$.

The only property (except $\Lambda \in P_{n}$ ) used in their proof is the following:

$$
\int_{S}|\Lambda|^{2 m} d \sigma \approx\left(\frac{1}{\sqrt{m}}\right)^{\delta(\Lambda)}
$$

The notation $\approx$ means that the ratios of two terms are bounded above and below. The only importance of the exponent $\delta(\Lambda)$ is the positivity. It is not hard to verify via Stirling's formula that (1) holds for every $\Lambda \in \Omega_{n}$. It follows that Ahern-Rudin's Paley-type gap theorems mentioned above hold for every $\Lambda \in \Omega_{n}$. The above observation leads to a natural conjecture that (1) holds for every $\Lambda \in P_{n}$. 
Now we return to the proof of Theorem 3.1. The following integral formula will be the main key to proving this. The normalized volume measure on $\mathbb{C}^{k}$ is denoted by $\nu_{k}$.

3.6. Proposition (Integration by Averaging). If $k$ and $l$ are positive integers such that $k+l=n$, then for every $f \in L^{1}\left(\sigma_{n}\right)$

$\int_{S_{n}} f d \sigma_{n}=\left(\begin{array}{c}n-1 \\ k\end{array}\right) \int_{B_{k}} \int_{S_{l}} f\left(z, \sqrt{1-|z|^{2}} \xi\right) d \sigma_{l}(\xi)\left(1-|z|^{2}\right)^{l-1} d \nu_{k}(z)$ holds.

Proof. Let $O$ be the group of all unitary transformations on $\mathbb{C}^{l}$ and fix $f \in L^{1}\left(\sigma_{n}\right)$. By Lemma 1.4.2 in [Ru] we have

$$
\int_{S_{n}} f d \sigma_{n}=\int_{S_{n}} \int_{O} f(\zeta, \mathscr{U} \eta) d \mathscr{U} d \sigma_{n}(\zeta, \eta)
$$

where $d \mathscr{U}$ is the Haar measure on $O$. In the right side of the above the inner integral is independent of $\eta$ once $\zeta$ is chosen, and hence by Formula 1.4.4 in [Ru] the left side of the above is equal to

$$
\left(\begin{array}{c}
n-1 \\
k
\end{array}\right) \int_{B_{k}} \int_{O} f\left(z, \sqrt{1-|z|^{2}} \mathscr{U} \xi\right) d \mathscr{U}\left(1-|z|^{2}\right)^{l-1} d \nu_{k}(z)
$$

where $\xi$ is an arbitrary point in $S_{l}$. By Proposition 1.4.7 in [Ru] the inner integral of the above is in turn equal to

$$
\int_{S_{l}} f\left(z, \sqrt{1-|z|^{2}} \xi\right) d \sigma_{l}(\xi)
$$

The proof is complete.

3.7. Proof of Theorem 3.1. We will assume $k \geq 2$ (in cases (a) and (b)) and $l \geq 2$. The proof when $l=1$ or $k=1$ is much simpler and in fact the proof given below can be repeated without any difficulty. $\psi$ will denote an arbitrary nonnegative Borel function on $[0,1)$. Also, $v_{i}$, $i=1,2$, denote the weights induced by $\phi_{i}$ and $w$ the weight induced by $\Lambda$. We will handle the constants $c=c\left(k, l, \phi_{1}, \phi_{2}\right)$ in the usual manner; they are not necessarily the same at any two occurrences.

Case (a). In this case $\operatorname{Max} \Lambda=\operatorname{Max} \phi_{1} \times \operatorname{Max} \phi_{2}$, topologically, and therefore $\delta(\Lambda)=\delta\left(\phi_{1}\right)+\delta\left(\phi_{2}\right)+1$. It remains to verify that

$$
w(r)=c(1-r)^{\left(\delta\left(\phi_{1}\right)+\delta\left(\phi_{2}\right)-1\right) / 2}[1+O(\sqrt{1-r})] \quad \text { as } r \uparrow 1 .
$$


Put $d=\operatorname{deg} \phi_{1}$ and $e=\operatorname{deg} \phi_{2}$. Note that $d, e \geq 1$. Define

$$
h(x)=b \sqrt{x^{d}(1-x)^{e}} \text { for } 0 \leq x \leq 1 .
$$

Note that $h$ is strictly increasing on $\left[0, x_{0}\right]$, strictly decreasing on $\left[x_{0}, 1\right]$, and $h\left(x_{0}\right)=1$ where $x_{0}=d /(d+e)$. Let $f$ be the inverse function of $h$ on $\left[0, x_{0}\right]$ and $g$ the inverse function of $h$ on $\left[x_{0}, 1\right]$.

Integration by averaging, integration in polar coordinates and change of variables show that

$$
\begin{aligned}
\int_{S_{n}} \psi \circ\left|\Lambda^{*}\right| d \sigma_{n}= & c \int_{0}^{1} x^{k-1}(1-x)^{l-1} \\
& \cdot \int_{S_{k}} \int_{S_{l}} \psi\left(h(x)\left|\phi_{1}(\xi)\right|\left|\phi_{2}(\eta)\right|\right) d \sigma_{l}(\eta) d \sigma_{k}(\xi) d x \\
= & c \int_{S_{k}} \int_{S_{l}} \int_{0}^{1} \theta(\rho) \psi\left(\rho\left|\phi_{1}(\xi)\right|\left|\phi_{2}(\eta)\right|\right) d \rho d \sigma_{l}(\eta) d \sigma_{k}(\xi)
\end{aligned}
$$

where $\theta=f^{k-1}(1-f)^{l-1} f^{\prime}-g^{k-1}(1-g)^{l-1} g^{\prime}$. By definitions of $v_{1}$ and $v_{2}$, the latter integral is equal to

$$
\begin{aligned}
& c \int_{0}^{1} \int_{0}^{1} \int_{0}^{1} \theta(\rho) \psi(\rho s t) d(\rho s t) v_{1}(s) v_{2}(t) d s d t \\
& \quad=c \int_{0}^{1} \psi(r) \iint_{s t \geq r} \theta_{s, t}(r) v_{1}(s) v_{2}(t) d s d t d r
\end{aligned}
$$

where $\theta_{s, t}(r)=\theta(r / s t)$ for $s t \geq r$. From this we conclude (see Remark 2.6)

$$
\begin{aligned}
w(r)= & c[1+O(\sqrt{1-r})] \\
& \times \iint_{s t \geq r} \theta_{s, t}(r)(1-s)^{\delta\left(\phi_{1}\right) / 2-1}(1-t)^{\delta\left(\phi_{2}\right) / 2-1} d s d t .
\end{aligned}
$$

It remains to estimate $\theta_{s, t}(r)$ as $r \uparrow 1$. Since $h^{\prime}\left(x_{0}\right)=0$ and $h^{\prime \prime}\left(x_{0}\right)<0$, we have

$$
h(x)=1+\frac{h^{\prime \prime}\left(x_{0}\right)}{2}\left(x-x_{0}\right)^{2}\left[1+O\left(\left|x-x_{0}\right|\right)\right] \text { as } x \rightarrow x_{0}
$$

and

$$
h^{\prime}(x)=h^{\prime \prime}\left(x_{0}\right)\left(x-x_{0}\right)\left[1+O\left(\left|x-x_{0}\right|\right)\right] \text { as } x \rightarrow x_{0} .
$$

From (3) and (4) it follows that

$$
\frac{1}{h^{\prime}(x)}=\frac{\left[1+O\left(\left|x-x_{0}\right|\right)\right]}{\sqrt{-2 h^{\prime \prime}\left(x_{0}\right)} \sqrt{1-h(x)}} \text { as } x \uparrow x_{0} \text {. }
$$


Putting $x=f(r)$, we obtain from (3) and (5) that

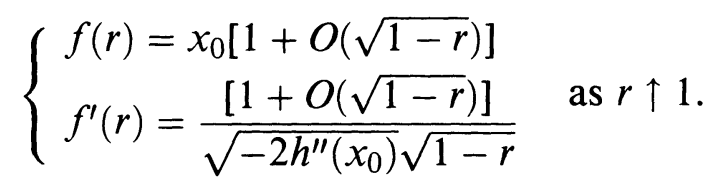

Similarly,

$$
\left\{\begin{array}{l}
g(r)=x_{0}[1+O(\sqrt{1-r})] \\
g^{\prime}(r)=-\frac{1+O(\sqrt{1-r})]}{\sqrt{-2 h^{\prime \prime}\left(x_{0}\right)} \sqrt{1-r}} \quad \text { as } r \uparrow 1 .
\end{array}\right.
$$

Hence

$$
\theta(r)=\frac{c[1+O(\sqrt{1-r})]}{\sqrt{1-r}} \text { as } r \uparrow 1
$$

and therefore

(6) $\quad \theta_{s, t}(r)=\frac{c[1+O(\sqrt{1-r})]}{\sqrt{s t-r}}$ uniformly in $s t \geq r$ as $r \uparrow 1$.

Insert (6) into (2) and estimate the double integral to obtain (1).

Cases (b) and (c). Let $d=\operatorname{deg} \Lambda$. As above, we have

$$
\begin{aligned}
& \int_{S_{n}} \psi \circ\left|\Lambda^{*}\right| d \sigma_{n} \\
&=c \int_{0}^{1} x^{k-1}(1-x)^{l-1} \\
& \cdot \int_{S_{k}} \int_{S_{l}} \psi\left(\left|a x^{d / 2} \phi_{1}(\xi)+(1-x)^{d / 2} \phi_{2}(\eta)\right|\right) d \sigma_{l}(\eta) d \sigma_{k}(\xi) d x \\
&= c \int_{0}^{1} x^{k-1}(1-x)^{l-1} \\
& \cdot \int_{U} \int_{U} \psi\left(\left|a x^{d / 2} \lambda+(1-x)^{d / 2} z\right|\right) v_{2}(z) d m(z) v_{1}(\lambda) d m(\lambda) d x \\
&= c \int_{0}^{1} x^{k-1}(1-x)^{l-1} \\
& \cdot \int_{0}^{1} \int_{\operatorname{Im} z \geq 0} \psi\left(\left|a x^{d / 2} s+(1-x)^{d / 2} z\right|\right) v_{2}(z) d m(z) s v_{1}(s) d s d x
\end{aligned}
$$

Compute the innermost integral in polar coordinates, make change of variables, and get

$$
\int_{0}^{1} \int_{-1}^{1} \frac{\psi\left(\sqrt{a^{2} x^{d} s^{2}+(1-x)^{d} t^{2}+2 a s t x^{d / 2}(1-x)^{d / 2} y}\right) d y t v_{2}(t) d t}{\sqrt{1-y^{2}}} \text {. }
$$


In the inner integral of the above we make another substitution

$$
r=\sqrt{a^{2} x^{d} s^{2}+(1-x)^{d} t^{2}+2 a s t x^{d / 2}(1-x)^{d / 2} y},
$$

simplify the integral obtained, and arrive at

$$
\int_{g_{s, l}(x)}^{h_{s, t}(x)} \frac{2 r \psi(r) d r}{\sqrt{r^{2}-g_{s, t}^{2}(x)} \sqrt{h_{s, t}^{2}(x)-r^{2}}}
$$

where

$$
\begin{aligned}
& h_{s, t}(x)=a s x^{d / 2}+t(1-x)^{d / 2} \text { and } \\
& g_{s, t}(x)=\left|a s x^{d / 2}-t(1-x)^{d / 2}\right| .
\end{aligned}
$$

So far,

$$
\begin{aligned}
& \int_{S_{n}} \psi \circ\left|\Lambda^{*}\right| d \sigma_{n} \\
&=c \int_{0}^{1} \int_{0}^{1}\left\{\int_{0}^{1} \int_{g_{s, t}(x)}^{h_{s, t}(x)} \frac{x^{k-1}(1-x)^{l-1} r \psi(r) d r d x}{\sqrt{r^{2}-g_{s, t}^{2}(x)} \sqrt{h_{s, t}^{2}(x)-r^{2}}}\right\} \\
& \cdot s v_{1}(s) t v_{2}(t) d s d t .
\end{aligned}
$$

For each fixed $s$ and $t$, the integral in the bracket is taken over the region $\left\{(x, r): g_{s, t}(x) \leq r \leq h_{s, t}(x)\right\}$ and therefore the interchange of the order of integration yields $\theta_{s, t}$ such that

$$
\int_{S_{n}} \psi \circ\left|\Lambda^{*}\right| d \sigma_{n}=c \int_{0}^{1} r \psi(r) \int_{0}^{1} \int_{0}^{1} \theta_{s, t}(r) s v_{1}(s) t v_{2}(t) d s d t d r .
$$

Accordingly,

$$
w(r)=c \int_{0}^{1} \int_{0}^{1} \theta_{s, t}(r) s v_{1}(s) t v_{2}(t) d s d t .
$$

Now we subdivide into two cases (b) and (c). Before going further, we assume $a>0$ (if $a=0$, then $\operatorname{Max} \Lambda=\operatorname{Max} \phi_{2}$, topologically, and the proof in case (a) can be easily adapted).

First, consider case (b). Since $d \geq 3, h_{s, t}$ is strictly convex for every $s$ and $t$. By elementary calculus if $t \geq(1 / 2)^{d / 2-1}$, then $h_{s, t}$ and $g_{s, t}$ are strictly decreasing on [0,1/3] for every $s$. For such $t$, let $\alpha_{s, t}$ and $\beta_{s, t}$ be the inverse functions of $h_{s, t}$ and $g_{s, t}$ on $[0,1 / 3]$, respectively. We first prove two sublemmas. 
Sublemma 1. Define for $t \geq r$ and $r \approx 1$

$$
E_{s, t}(r)=\int_{\beta_{s, l}(r)}^{\alpha_{s, t}(r)} \frac{x^{k-1}(1-x)^{l-1} d x}{\sqrt{r^{2}-g_{s, t}^{2}(x)} \sqrt{h_{s, t}^{2}(x)-r^{2}}} .
$$

Then $E_{s, t}(r)=c(t-r)^{k-1}[1+O(\sqrt{1-r})]$ uniformly in $t \geq r$ and $s$ as $r \uparrow 1$.

Proof of Sublemma 1. We easily obtain

$$
\left\{\begin{array}{l}
d \alpha_{s, t}(r)=2(t-r)[1+O(\sqrt{1-r})], \\
d \beta_{s, t}(r)=2(t-r)[1+O(\sqrt{1-r})], \\
\alpha_{s, t}(r)=\beta_{s, t}(r)[1+O(\sqrt{1-r})],
\end{array}\right.
$$

where $O(\sqrt{1-r})$ is uniform in $t \geq r$ and $s$ as $r \uparrow 1$.

Define $H_{s, t}=h_{s, t}^{2}$ and $G_{s, t}=g_{s, t}^{2}$. Since $d \geq 3, H_{s, t}^{\prime}(x)=$ $-d t^{2}[1+O(\sqrt{x})]$ uniformly in $t \approx 1$ and $s$ as $x \downarrow 0$. Thus by the mean value theorem and (8), if $\beta_{s, t}(r)<x<\alpha_{s, t}(r)$, then

$$
H_{s, t}(x)-r^{2}=d\left(\alpha_{s, t}(r)-x\right)[1+O(\sqrt{1-r})]
$$

uniformly in $t \geq r, s$ and $x \in\left(\beta_{s, t}(r), \alpha_{s, t}(r)\right)$ as $r \uparrow 1$. Similarly,

$$
r^{2}-G_{s, t}(x)=d\left(x-\beta_{s, t}(r)\right)[1+O(\sqrt{1-r})]
$$

uniformly in $t \geq r, s$ and $x \in\left(\beta_{s, t}(r), \alpha_{s, t}(r)\right)$ as $r \uparrow 1$. It follows that

$$
E_{s, t}(r)=c[1+O(\sqrt{1-r})] \int_{\beta_{s, t}(r)}^{\alpha_{s, t}(r)} \frac{x^{k-1}(1-x)^{l-1} d x}{\sqrt{x-\beta_{s, t}(r)} \sqrt{\alpha_{s, t}(r)-x}}
$$

uniformly in $t \geq r$ and $s$ as $r \uparrow 1$. In this integral, make the substitution

$$
y=\frac{x-\beta_{s, t}(r)}{\alpha_{s, t}(r)-\beta_{s, t}(r)}
$$

and get

$$
\begin{aligned}
& \int_{0}^{1}\left(\left[\alpha_{s, t}(r)-\beta_{s, t}(r)\right] y+\beta_{s, t}(r)\right)^{k-1} \\
& \quad \cdot\left(1-\beta_{s, t}(r)+\left[\beta_{s, t}(r)-\alpha_{s, t}(r)\right] y\right)^{l-1} \frac{d y}{\sqrt{y(1-y)}} .
\end{aligned}
$$

This shows the sublemma by (8).

If $a=1$ and if $s \geq(1 / 2)^{d / 2-1}$, then $h_{s, t}$ and $g_{s, t}$ are strictly increasing on $[2 / 3,1]$ for every $t$. For such $s$, let $\gamma_{s, t}$ and $\delta_{s, t}$ be the inverse functions of $h_{s, t}$ and $g_{s, t}$ (with $a=1$ ) on [2/3,1], respectively. The proof of the following sublemma is "symmetric" and is omitted. 
Sublemma 2. Define for $s \geq r$ and $r \approx 1$

$$
F_{s, t}(r)=\int_{\gamma_{s, t}(r)}^{\delta_{s, t}(r)} \frac{x^{k-1}(1-x)^{l-1} d x}{\sqrt{r^{2}-g_{s, t}^{2}(x)} \sqrt{h_{s, t}^{2}(x)-r^{2}}} .
$$

Then $F_{s, t}(r)=c(s-r)^{l-1}[1+O(\sqrt{1-r})]$ uniformly in $s \geq r$ and $t$ as $r \uparrow 1$.

Assume $a=1$. Then $\operatorname{Max} \Lambda$ is the disjoint union of $\operatorname{Max} \phi_{1}$ and $\operatorname{Max} \phi_{2}$. Hence it is enough to prove that

$$
\begin{aligned}
w(r)=[ & \left.c(1-r)^{\delta\left(\phi_{1}\right) / 2+l-1}+c(1-r)^{\delta\left(\phi_{2}\right) / 2+k-1}\right] \\
& \cdot[1+O(\sqrt{1-r})] \quad \text { as } r \uparrow 1 .
\end{aligned}
$$

By a geometric consideration, for $r$ sufficiently close to 1 ,

$$
\theta_{s, t}(r)= \begin{cases}0 & \text { if } t \leq r \text { and } s \leq r, \\ E_{s, t}(r) & \text { if } s \leq r \leq t, \\ F_{s, t}(r) & \text { if } t \leq r \leq s, \\ E_{s, t}(r)+F_{s, t}(r) & \text { if } r \leq t \text { and } r \leq s .\end{cases}
$$

Hence by (7), up to a constant factor,

$$
\begin{aligned}
w(r)= & \int_{r}^{1} \int_{0}^{r} E_{s, t}(r) s v_{1}(s) d s t v_{2}(t) d t \\
& +\int_{0}^{r} \int_{r}^{1} F_{s, t}(r) s v_{1}(s) d s t v_{2}(t) d t \\
& +\int_{r}^{1} \int_{r}^{1}\left(E_{s, t}(r)+F_{s, t}(r)\right) s v_{1}(s) d s t v_{2}(t) d t \\
= & \int_{r}^{1} \int_{0}^{1} E_{s, t}(r) s v_{1}(s) d s t v_{2}(t) d t \\
& +\int_{0}^{1} \int_{r}^{1} F_{s, t}(r) s v_{1}(s) d s t v_{2}(t) d t=\mathrm{I}+\mathrm{II} .
\end{aligned}
$$

By Sublemma 1

$$
\begin{aligned}
\mathrm{I} & =c[1+O(\sqrt{1-r})] \int_{r}^{1}(t-r)^{k-1}(1-t)^{\delta\left(\phi_{2}\right) / 2-1} d t \\
& =c(1-r)^{\delta\left(\phi_{2}\right) / 2+k-1}[1+O(\sqrt{1-r})] \quad \text { as } r \uparrow 1 .
\end{aligned}
$$

Similarly, by Sublemma 2

$$
\mathrm{II}=c(1-r)^{\delta\left(\phi_{1}\right) / 2+l-1}[1+O(\sqrt{(1-r})] \quad \text { as } r \uparrow 1 .
$$

This proves (9). 
Now assume $a<1$. Then $\operatorname{Max} \Lambda=\operatorname{Max} \phi_{2}$, topologically. Thus we need show

$$
w(r)=c(1-r)^{\delta\left(\phi_{2}\right) / 2+k-1}[1+O(\sqrt{1-r})] \quad \text { as } r \uparrow 1 .
$$

As above, for $r$ sufficiently close to 1 ,

$$
\theta_{s, t}(r)= \begin{cases}0 & \text { if } t \leq r \\ E_{s, t}(r) & \text { if } r \leq t\end{cases}
$$

Thus by (7) and Sublemma 1

$$
\begin{aligned}
w(r) & =c \int_{r}^{1} \int_{0}^{1} E_{s, t}(r) s v_{1}(s) d s t v_{2}(t) d t \\
& =c[1+O(\sqrt{1-r})] \int_{r}^{1}(t-r)^{k-1}(1-t)^{\delta\left(\phi_{2}\right) / 2-1} d t \quad \text { as } r \uparrow 1 .
\end{aligned}
$$

After a little calculation we obtain (10).

Finally, we consider case (c). In this case $k=1$ and $l=n-1$. Also, $\operatorname{Max} \Lambda=\operatorname{Max} \phi_{2}$, topologically. Thus we need verify

$$
w(r)=c(1-r)^{\delta\left(\phi_{2}\right) / 2}[1+O(\sqrt{1-r})] \quad \text { as } r \uparrow 1 .
$$

Note that (7) is still available with $s=1$, i.e.,

$$
w(r)=c \int_{0}^{1} \theta_{1, t}(r) t v_{2}(t) d t
$$

For $r>a$,

$$
\theta_{1, t}(r)= \begin{cases}0 & \text { if } t \leq r, \\ E_{t}(r) & \text { if } r \leq t,\end{cases}
$$

where $E_{t}(r)$ is the function as in Sublemma 1 with appropriately defined functions. More precisely, for $t \geq r>a$,

$$
\begin{array}{llrl}
h_{t}(x) & =a x+t(1-x), & g_{t}(x) & =|a x-t(1-x)|, \\
\alpha_{t}(r) & =\frac{t-r}{t-a}, & \beta_{t}(r) & =\frac{t-r}{t+a}
\end{array}
$$

and

$$
E_{t}(r)=\int_{\beta_{t}(r)}^{\alpha_{t}(r)} \frac{(1-x)^{n-2} d x}{\sqrt{r^{2}-g_{t}^{2}(x)} \sqrt{h_{t}^{2}(x)-r^{2}}} .
$$

By (11) and (12), for $r>a$,

$$
w(r)=c[1+O(\sqrt{1-r})] \int_{r}^{1} E_{t}(r)(1-t)^{\delta\left(\phi_{2}\right) / 2-1} d t \quad \text { as } r \uparrow 1 .
$$


Thus it suffices to show that

$$
E_{t}(r)=c[1+O(\sqrt{1-r})] \text { uniformly in } t \geq r \text { as } r \uparrow 1 \text {. }
$$

Clearly $\alpha_{t}(r)=O(1-r)$ and $\beta_{t}(r)=O(1-r)$ uniformly in $t \geq r$ as $r \uparrow 1$. Hence it is not hard to see that

$$
h_{t}^{2}(x)-r^{2}=2(1-a)\left(\alpha_{t}(r)-x\right)[1+O(1-r)]
$$

and

$$
r^{2}-g_{t}^{2}(x)=2(1+a)\left(x-\beta_{t}(r)\right)[1+O(1-r)]
$$

uniformly in $t \geq r$ and $x \in\left(\beta_{t}(r), \alpha_{t}(r)\right)$ as $r \uparrow 1$. Substitute these into (13) and obtain (14) as before.

\section{REFERENCES}

[A] P. Ahern, On the behavior near torus of functions holomorphic in the ball, Pacific J. Math., 107 (1983), 267-278.

[AR1] P. Ahern and W. Rudin, Bloch functions, BMO and boundary zeros, Indiana Univ. Math. J., 36 (1987), 131-148.

[AR2] - Paley-type gap theorems for $H^{p}$-functions on the ball, Indiana Univ. Math. J., to appear.

[C] B. R. Choe, Cauchy integral equalities and applications, Trans. Amer. Math. Soc., to appear.

[F1] H. Federer, Curvature measures, Trans. Amer. Math. Soc., 93 (1959), 418491.

[F2] , Geometric Measure Theory, Springer-Verlag, Berlin, Heidelberg, New York, 1969.

[G] F. R. Gantmacher, The Theory of Matrices, Vol. I, Chelsea, 1959.

[Ru] W. Rudin, Function Theory in the Unit Ball of $\mathbb{C}^{n}$, Springer-Verlag, Berlin, Heidelberg, New York, 1980.

[Rus] P. Russo, Boundary behavior of $B M O\left(B_{n}\right)$, Trans. Amer. Math. Soc., 292, (1985), 733-740.

[S] N. Sibony, Valeurs au bord de fonctions holomorphes et ensembles polynomialement convexes, Lecture Notes in Mathematics, No. 578, pp. 300-313, Springer-Verlag, Heidelberg, 1977.

Received December 17, 1987.

Michigan State University

EAST LANSING, MI 48824 


\section{PACIFIC JOURNAL OF MATHEMATICS EDITORS}

\author{
V. S. VARADARAJAN \\ (Managing Editor) \\ University of California \\ Los Angeles, CA 90024-1555-05 \\ Herbert Clemens \\ University of Utah \\ Salt Lake City, UT 84112 \\ THOMAS ENRIGHT \\ University of California, San Diego \\ La Jolla, CA 92093
}

\author{
R. FINN \\ Stanford University \\ Stanford, CA 94305 \\ Hermann Flaschka \\ University of Arizona \\ Tucson, AZ 85721 \\ VAUGHAN F. R. JoNES \\ University of California \\ Berkeley, CA 94720
}

SteVen KerCKHOFF

Stanford University

Stanford, CA 94305

\author{
RoBION KIRBY \\ University of California \\ Berkeley, CA 94720 \\ C. C. MOORE \\ University of California \\ Berkeley, CA 94720
}

HAROLD STARK

University of California, San Diego

La Jolla, CA 92093

\section{ASSOCIATE EDITORS}
R. ARENS
E. F. BECKENBACH
B. H. NEUMANN
F. WoLf
K. Yoshida

(1906-1982)

\section{SUPPORTING INSTITUTIONS}

UNIVERSITY OF ARIZONA

UNIVERSITY OF BRITISH COLUMBIA

CALIFORNIA INSTITUTE OF TECHNOLOGY

UNIVERSITY OF CALIFORNIA

MONTANA STATE UNIVERSITY

UNIVERSITY OF NEVADA, RENO

NEW MEXICO STATE UNIVERSITY

OREGON STATE UNIVERSITY

\author{
UNIVERSITY OF OREGON \\ UNIVERSITY OF SOUTHERN CALIFORNIA \\ STANFORD UNIVERSITY \\ UNIVERSITY OF HAWAII \\ UNIVERSITY OF TOKYO \\ UNIVERSITY OF UTAH \\ WASHINGTON STATE UNIVERSITY \\ UNIVERSITY OF WASHINGTON
}

The Supporting Institutions listed above contribute to the cost of publication of this Journal, but they are not owners or publishers and have no responsibility for its content or policies.

Mathematical papers intended for publication in the Pacific Journal of Mathematics should be in typed form or offset-reproduced (not dittoed), double spaced with large margins. Please do not use built up fractions in the text of the manuscript. However, you may use them in the displayed equations. Underline Greek letters in red, German in green, and script in blue. The first paragraph must be capable of being used separately as a synopsis of the entire paper. In particular it should contain no bibliographic references. Please propose a heading for the odd numbered pages of less than 35 characters. Manuscripts, in triplicate, may be sent to any one of the editors. Please classify according to the scheme of Math. Reviews, Index to Vol. 39. Supply name and address of author to whom proofs should be sent. All other communications should be addressed to the managing editor, or Elaine Barth, University of California, Los Angeles, California 90024-1555-05.

There are page-charges associated with articles appearing in the Pacific Journal of Mathematics. These charges are expected to be paid by the author's University, Government Agency or Company. If the author or authors do not have access to such Institutional support these charges are waived. Single authors will receive 50 free reprints; joint authors will receive a total of 100 free reprints. Additional copies may be obtained at cost in multiples of 50 .

The Pacific Journal of Mathematics is issued monthly as of January 1966. Regular subscription rate: $\$ 190.00$ a year (5 Vols., 10 issues). Special rate: $\$ 95.00$ a year to individual members of supporting institutions.

Subscriptions, orders for numbers issued in the last three calendar years, and changes of address should be sent to Pacific Journal of Mathematics, P.O. Box 969, Carmel Valley, CA 93924, U.S.A. Old back numbers obtainable from Kraus Periodicals Co., Route 100, Millwood, NY 10546.

The Pacific Journal of Mathematics at P.O. Box 969, Carmel Valley, CA 93924 (ISSN 0030-8730) publishes 5 volumes per year. Application to mail at Second-class postage rates is pending at Carmel Valley, California, and additional mailing offices. Postmaster: send address changes to Pacific Journal of Mathematics, P.O. Box 969, Carmel Valley, CA 93924.

PUBLISHED BY PACIFIC JOURNAL OF MATHEMATICS, A NON-PROFIT CORPORATION Copyright (C) 1989 by Pacific Journal of Mathematics 


\section{Pacific Journal of Mathematics}

Vol. 139, No. $2 \quad$ June, 1989

Joseph Barback and William Jackson, On recursive functions and regressive isols .................................... 209

Boo Rim Choe, Weights induced by homogeneous polynomials .........225

Caroline Galt Grant, Hyperbolicity of surfaces modulo rational and elliptic curves

Christian Ulrik Jensen and Birge Huisgen-Zimmermann, Algebraic

compactness of ultrapowers and representation type $\ldots \ldots \ldots \ldots \ldots 251$

Douglas S. Kurtz, Operator estimates using the sharp function . .........267

Patrick Thomas Perkins, Commutative subalgebras of the ring of

differential operators on a curve

Christopher Skinner, The Diophantine equation $x^{2}=4 q^{n}-4 q+1 \ldots \ldots 303$

Wilhelm F. Stoll, On the propagation of dependences $\ldots \ldots \ldots \ldots \ldots \ldots 311$

Ernest A. Thieleker, A unitary representation of the conformal group on

Minkowski space and dynamical groups. I

Roger Craig Zierau, A construction of harmonic forms on

$U(p+1, q) / U(p, q) \times U(1)$ 